\title{
Nuclear receptor $4 A 1$ as a drug target for breast cancer chemotherapy
}

\author{
Erik Hedrick, Syng-Ook Lee', Ravi Doddapaneni ${ }^{2}$, Mandip Singh ${ }^{2}$ \\ and Stephen Safe
}

Department of Veterinary Physiology and Pharmacology, Texas A\&M University, 4466 TAMU, College Station, Texas 77843-4466, USA

${ }^{1}$ Department of Food Science and Technology, Keimyung University, Daegu 704701, Republic of Korea ${ }^{2}$ Department of Pharmaceutics, College of Pharmacy and Pharmaceutical Sciences, Florida A\&M University, Tallahassee, Florida 32307, USA
Correspondence should be addressed to S Safe or M Singh Emails

ssafe@cvm.tamu.edu or mandip.sacheva@gmail.com

\begin{abstract}
The orphan nuclear receptor 4A1 (NR4A1) is overexpressed in mammary tumors and breast cancer cell lines. The functional activity of this receptor was investigated by RNA interference with oligonucleotides targeted to NR4A1 (siNR4A1) and by treatment with NR4A1 antagonists. Breast cancer cells were treated with NR4A1 antagonists or transfected with siNR4A. Effects on cell proliferation and apoptosis as well as specific genes associated with these responses were investigated in MCF-7, SKBR3, and MDA-MB-231 cells, and in athymic nude mice bearing MDA-MB-231 cells as xenografts. Transfection of MCF-7, MDAMB-231, and SKBR3 breast cancer cells with siNR4A1 decreased cell proliferation and induced apoptosis in these cell lines. Transfection of breast cancer cells with siNR4A1 also decreased expression of Sp-regulated genes including survivin, bcl-2, and epidermal growth factor receptor, inhibited mTOR signaling in MCF-7 cells that express WT p53, and activated oxidative and endoplasmic reticulum stress through downregulation of thioredoxin domain-containing 5 and isocitrate dehydrogenase 1. 1,1-Bis(3'-indolyl)-1-( $p$-substituted phenyl)methanes (C-DIMs) are NR4A1 ligands that act as NR4A1 antagonists. Treatment with selected analogs also inhibited breast cancer cell and tumor growth and induced apoptosis. The effects of C-DIM/NR4A1 antagonists were comparable to those observed after NR4A1 knockdown. Results with siNR4A1 or C-DIMs/NR4A1 antagonists in breast cancer cells and tumors were similar to those previously reported in pancreatic, lung, and colon cancer cells. They demonstrate the potential clinical applications of NR4A1 antagonists in patients with tumors that overexpress this receptor.
\end{abstract}
Key Words
- NR4A1
- indole derivative
antagonists

Endocrine-Related Cancer (2015) 22, 831-840

\section{Introduction}

Nuclear receptor 4A1 (NR4A1, Nur77, TR3) is a member of the NR4A orphan receptor sub-family of nuclear receptors. NR4A receptors (NR4A1, NR4A2, and NR4A3) play essential roles in metabolic processes, inflammation, vascular function, steroidogenesis, and the CNS (Maxwell \& Muscat 2006,
Pearen \& Muscat 2010, Lee et al. 2011). NR4A1 is overexpressed in multiple tumors and cancer cell lines. Results of receptor knockdown by RNA interference (RNAi) demonstrate that in solid tumors the receptor is pro-oncogenic and regulates cell growth and survival (Uemura \& Chang 1998, 
Bras et al. 2000, Kolluri et al. 2003, Zeng et al. 2006, Lee et al. 2010, 2011, 2012, 2014a, Wu et al. 2011). Several proapoptotic agents including phorbol esters and adamantylderived retinoids induce expression and nuclear export of NR4A1 which subsequently binds mitochondrial bcl-2 to form a pro-apoptotic complex that decreases mitochondrial membrane potential (Li et al. 2000, Lin et al. 2004, Zhang 2007). This has led to the development of peptide mimics that convert bcl-2 into an apoptotic complex; similar results have been reported for the taxane-derived anticancer agent paclitaxel (Kolluri et al. 2008, Ferlini et al. 2009). Cytosporone $\mathrm{B}$ and related analogs have been identified as ligands for NR4A1 (Zhan et al. 2008, Liu et al. 2010), and two additional compounds, (ethyl 2-(2,3,4-trimethoxy-6-(1-octanoyl)phenyl)acetate and 1-(3,4,5-trihydroxyphenyl) no-nan-1-one), also bind NR4A1 (Zhan et al. 2012, Wang et al. 2014). Ethyl 2-(2,3,4-trimethoxy-6-(1-oct-anoyl) phenyl)acetate inactivates nuclear NR4A1, whereas 1-(3,4,5-trihydroxyphenyl)no-nan-1-one and cytosporone B induce nuclear export of NR4A1.

Studies in this laboratory have been investigating a series of 1,1-bis(3'-indolyl)-1-( $p$-substituted phenyl) methane (C-DIM) analogs and their effects on NR4A1 and NR4A1-dependent transactivation (Chintharlapalli et al. 2005, Lee et al. 2009, 2010, 2012, 2014a,b, Cho et al. 2010). Since NR4A1 exhibits pro-oncogenic activity, we have been focused on the identification of C-DIMs that inactivate NR4A1. The $p$-hydroxyphenyl analog (DIM-C-pPhOH) was characterized as a compound that inactivated nuclear NR4A1 in cancer cell lines but this was not accompanied by nuclear export of NR4A1 (Lee et al. 2010, 2012, 2014a). Subsequent studies comparing the effects of DIM-C-pPhOH and knockdown of NR4A1 (siNR4A1) by RNAi identified three major pro-oncogenic pathways and associated genes regulated by NR4A1 that were inhibited by DIM-C-pPhOH: i) NR4A1 regulates expression of genes such as survivin through interactions with specificity protein 1 (Sp1) bound to their proximal GC-rich promoters (Lee et al. 2010); ii) NR4A1 inactivates p53 to enhance mTOR signaling in lung and colon cancer cells expressing WT p53 (Lee et al. 2012, 2014b); and iii) NR4A1 regulates expression of thioredoxin domain-containing 5 (TXNDC5) and isocitrate dehydrogenase 1 (IDH1) to maintain low levels of oxidative stress (Lee et al. 2014a; Fig. 1A).

Recent studies show that NR4A1 is overexpressed in ER-positive and ER-negative breast tumors (Muscat et al. 2013). NR4A1 expression in breast tumors is correlated with decreased relapse-free survival (Zhou et al. 2014). Results of NR4A1 overexpression in breast cancer cells suggest that NR4A1 may be anti-migratory (Alexopoulou

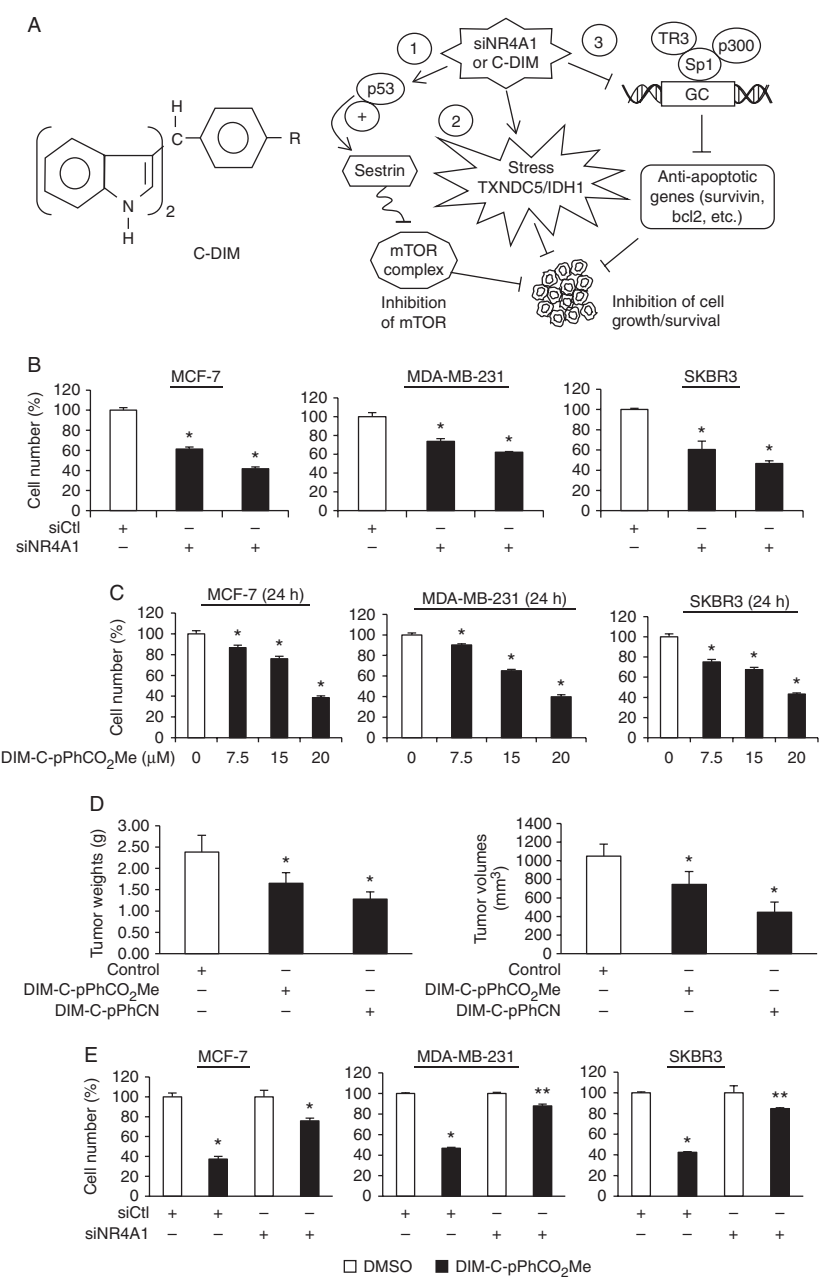

Figure 1

NR4A1-regulated pathways and effects of NR4A1 knockdown on breast cancer cell proliferation. (A) NR4A1-regulated pathways/genes that can be targeted by C-DIM/NR4A1 antagonists. (B) Cells were transfected with two siNR4A1 oligonucleotides ( 1 and 2 ) and cell numbers were determined after $72 \mathrm{~h}$. (C) Cells were treated with different concentrations of DIM-C-pPhCO ${ }_{2} \mathrm{Me}$ for $24 \mathrm{~h}$ and the number of cells were then determined. (D) Athymic nude mice bearing MDA-MB-231 cells (orthotopic) were administered corn oil (control), DIM-C-pPhCO 2 Me or DIM-C-pPhCN ( $50 \mathrm{mg} / \mathrm{kg}$ per day) by oral gavage for 28 days, and effects on tumor growth and weight were determined (significantly decreased; ${ }^{*} P<0.01$ ). (E) Cells were transfected with siCtl (non-specific oligonucleotide) or siNR4A1 and then treated with $20 \mu \mathrm{M}$ DIM-C-pPhCO ${ }_{2} \mathrm{Me}$ for $24 \mathrm{~h}$ and the number of cells were then counted. The control (untreated) groups (set at 100\%) in studies on the growth inhibitory effects of C-DIMs after knockdown are cells transfected with siNR4A1 and treated with DMSO. Results (B, C and D) are means \pm S.E.M. for at least three separate determinations for each treatment group. Significant $(P<0.05)$ growth inhibition is indicated $\left(^{*}\right)$ and a significant decrease in the growth inhibitory effects of DIM-C-pPhCO ${ }_{2} \mathrm{Me}$ after NR4A1 knockdown is also indicated (**).

et al. 2010). However, a recent study reported pro-migratory activity for this receptor (Zhou et al. 2014). Research in this laboratory has demonstrated pro-oncogenic functions of NR4A1 in pancreatic, colon, and lung cancer cells. This

Published by Bioscientifica Ltd 
study investigates the functions of this receptor in breast cancer cells and the effects of C-DIM/NR4A1 antagonists. The results clearly demonstrate the pro-oncogenic functions of NR4A1 in breast cancer and also demonstrate that C-DIM/NR4A1 antagonists represent a potential novel approach for treating breast cancer patients that overexpress this orphan receptor.

\section{Materials and methods}

\section{Cell lines and antibodies}

MCF-7, MDA-MB-231, and SKBR3 human breast cancer cell lines were purchased from American Type Culture Collection (Manassas, VA, USA) and were kept frozen until initiation of these studies. The cells were received at low passage $(<15)$ and new frozen stocks were used every 6-8 weeks. The three cell lines were authenticated by Biosynthesis (Lewisville, TX, USA) on 3rd February 2015. Cells were maintained at $37^{\circ} \mathrm{C}$ in the presence of $5 \% \mathrm{CO}_{2}$ in DMEM/Ham's F-12 medium with 10\% fetal bovine serum with antibiotic. $\beta$-actin antibody and DMEM were purchased from Sigma-Aldrich. Sp1 antibody was purchased from Millipore (Temecula, CA, USA). Caspases 7 and 8, sestrin 2 (SESN2), bcl2, CHOP, ATF4, IDH1, IRE, ATF6, GRP78, and epidermal growth factor receptor (EGFR) antibodies were purchased from Santa Cruz Biotechnologies. Caspase 3, cleaved poly ADP ribose polymerase (c-PARP; 9541), phospho mTOR, mTOR, phospho AMPK $\alpha$, AMPK $\alpha$, phospho p70S6K, p70S6K, phospho S6RP, S6RP, phospho 4EBP1, 4EBP1, and survivin antibodies were purchased from Cell Signaling Technologies (Danvers, MA, USA). TXNDC5 antibody was purchased from Genetex (Irvine, CA, USA). XBP-1s and phospho PERK were obtained from Biolegend (San Diego, CA, USA). The Apoptotic, Necrotic, and Healthy Cells Quantification Kit was purchased from Biotium (Hayward, CA, USA). Cells were visualized under an EVOS fl, Fluorescence microscope, from Advanced Microscopy Group using a multiband filter set for FITC, rhodamine, and DAPI. The C-DIM compounds were prepared and immunostaining for NR4A1 was carried out as previously described (Lee et al. 2010, 2012, 2014a).

\section{Cell proliferation assay}

MCF-7, MDA-MB-231, and SKBR3 breast cancer cells $\left(1.0 \times 10^{5} /\right.$ well $)$ were plated in 12 -well plates and allowed to attach for $24 \mathrm{~h}$. Cells were treated with 1,1-bis(3'-indolyl)1-(p-carboxymethylphenyl)methane (DIM-C-pPhCO ${ }_{2} \mathrm{Me}$ ) in DMSO for 24 or $48 \mathrm{~h}$ or transfected with siNR4A1 or
iGL2 (control siRNA) in lipofectamine for $72 \mathrm{~h}$. Cells were then trypsinized and counted using a Coulter Z1 cell counter and growth inhibition was determined. Each experiment was carried out in triplicate and results were expressed as the mean \pm s.E.M. for each set of experiments. Cells were also treated with C-DIMs after NR4A1 knockdown.

\section{Annexin V staining}

MCF-7, MDA-MB-231, and SKBR3 cells $\left(1.0 \times 10^{5} /\right.$ well $)$ were seeded in two-well Nunc Lab-Tek chambered B\#1.0 Borosilicate coverglass slides from Thermo Scientific (Waltham, MA, USA) and were allowed to attach for $24 \mathrm{~h}$. The medium was then changed to DMEM/Ham F-12 medium containing $2.5 \%$ charcoal-stripped fetal bovine serum, and either DMSO or DIM-C-pPhCO $2 \mathrm{Me}(15 \mu \mathrm{M})$ was added for $24 \mathrm{~h}$. For siRNA treatment, cells were transfected with iGL2 or $100 \mathrm{~nm}$ siNR4A1 (1 or 2) for $72 \mathrm{~h}$. Apoptosis was analyzed by Apoptotic and Necrotic Assay Kit (Biotium), which contained FITC-Annexin V, ethidium homodimer III, and Hoechst 3342. Apoptosis, Necrotic, and Healthy Cell Detection Kit was used according to the manufacturer's protocol and cells were visualized under an EVOS fl, fluorescence microscope, from Advanced Microscopy. The proportion of apoptotic cells was determined by the amount of green fluorescence observed in the treatment groups relative and normalized to the control group.

\section{Western blot analysis}

Breast cancer cells $\left(3.0 \times 10^{5} /\right.$ well $)$ were seeded in DMEM/ Ham's F-12 medium in six-well plates. Cells were allowed to attach for $24 \mathrm{~h}$ and treated with varying concentrations of DIM-C-pPhCO ${ }_{2} \mathrm{Me}$ for $24 \mathrm{~h}$ or with $100 \mathrm{~nm}$ of siNR4A1 for $72 \mathrm{~h}$. Cells were lysed with high salt lysis buffer (with protease inhibitor cocktail) and quantitated with a Bradford reagent. Lysates were then analyzed by SDS-PAGE and transferred onto a PVDF membrane by wet electroblotting. Membranes were then incubated with a primary antibody, followed by a secondary antibody. Western blot analysis was determined as described and Immobilon western chemiluminescence substrates (Millipore) were used to develop images captured on a Kodak 4000 MM Pro image station (Molecular Bioimaging, Bend, OR, USA).

\section{siRNAi assay}

Breast cancer cells were seeded $\left(1.2 \times 10^{5} /\right.$ well $)$ in six-well plates in DMEM/Ham's F-12 medium supplemented with $2.5 \%$ charcoal-stripped fetal bovine serum and left to

Published by Bioscientifica Ltd. 
attach for $24 \mathrm{~h}$. Knockdown of NR4A1 was carried out using Lipofectamine 2000 reagent according to the manufacturer's protocol. Small inhibitory RNAs and GL2 (nonspecific oligonucleotide) were prepared and purchased from Sigma-Aldrich. The siRNA complexes used in the study are as follows: siGL2-5'-CGU ACG CGG AAU ACU UCG A-3' ; siNR4A1 (1)-SASI_Hs02_00333289; siNR4A1 (2)SASI_Hs01_00182072.

\section{Generation and measurement of reactive oxygen species (ROS)}

Cellular ROS levels were ascertained using the cell permeable probe CM- $\mathrm{H}_{2}$ DCFDA (5-(and-6)-chloromethyl$2^{\prime} 7^{\prime}$-dichlorodihydrofluorescein diacetate acetyl ester) from Invitrogen. Following treatment of the cells for 12 or $24 \mathrm{~h}$ with DIM-C-pPHCO ${ }_{2} \mathrm{Me}$ or siNR4A1 for $72 \mathrm{~h}$, cells that were plated on a six-well culture plate were trypsinized, neutralized, then loaded with $10 \mu \mathrm{M}$ of probe for $20 \mathrm{~min}$, and washed once with serum free medium. ROS was then measured by flow cytometry using Accuri's C6 Flow Cytometer (Accuri, Ann Arbor, MI, USA).

\section{Triple negative breast cancer (TBNC) orthotopic xenograft model}

Female BALB/c nude mice (6-8 weeks old) were obtained (Charles River Laboratory, Wilmington, MA, USA) and maintained under specific pathogen-free conditions, housed in isolated vented cages and allowed to acclimate for 1 week with a standard chow diet. The animals were housed at Florida A\&M University in accordance with the standards of the Guide for the Care and Use of Laboratory Animals and the Association for Assessment and Accreditation of Laboratory Animal Care (AAALAC). The protocol of the animal study was approved by the Institutional Animal Care and Use Committee (IACUC), Florida A\&M University, FL, USA. MDA-MB-231 cells $\left(1 \times 10^{6}\right.$ cells) were detached, resuspended in $100 \mu \mathrm{l}$ of PBS with matrigel (BD Bioscience, Bedford, MA, USA), and implanted subcutaneously in the mammary fat pad of mice. When tumors reached about 40$50 \mathrm{~mm}^{3}$ in size, the animals were randomized into control and treatment groups (six animals per group) and mice were treated with placebo or DIM-C-pPhCO ${ }_{2} \mathrm{Me}$ or 1,1-bis(3'indolyl)-1-(p-cyanophenyl)methane (DIM-C-pPhCN) (50 $\mathrm{mg} / \mathrm{kg}$ per day) in nano liquid carrier (administered in sodium carboxymethyl cellulose) by oral gavage every 2nd day for 4 weeks. Tumor volumes and weights, and body weight were determined. The tumor size was measured using Vernier calipers, and the tumor volume was estimated by the formula: tumor volume $\left(\mathrm{mm}^{3}\right)=(L \times$ $\left.W^{2}\right) \times \frac{1}{2}$, where $L$ is the length and $W$ is the width of the tumor. Tumor lysates were obtained and analyzed for protein expression by western blots.

\section{Statistical analysis}

Statistical significance of differences between the treatment groups was determined by Student's $t$-test. The results are expressed as means with error bars representing 95\% CIs for three experiments for each group unless otherwise indicated. A $P$ value $<0.05$ was considered statistically significant. All statistical tests were two-sided.

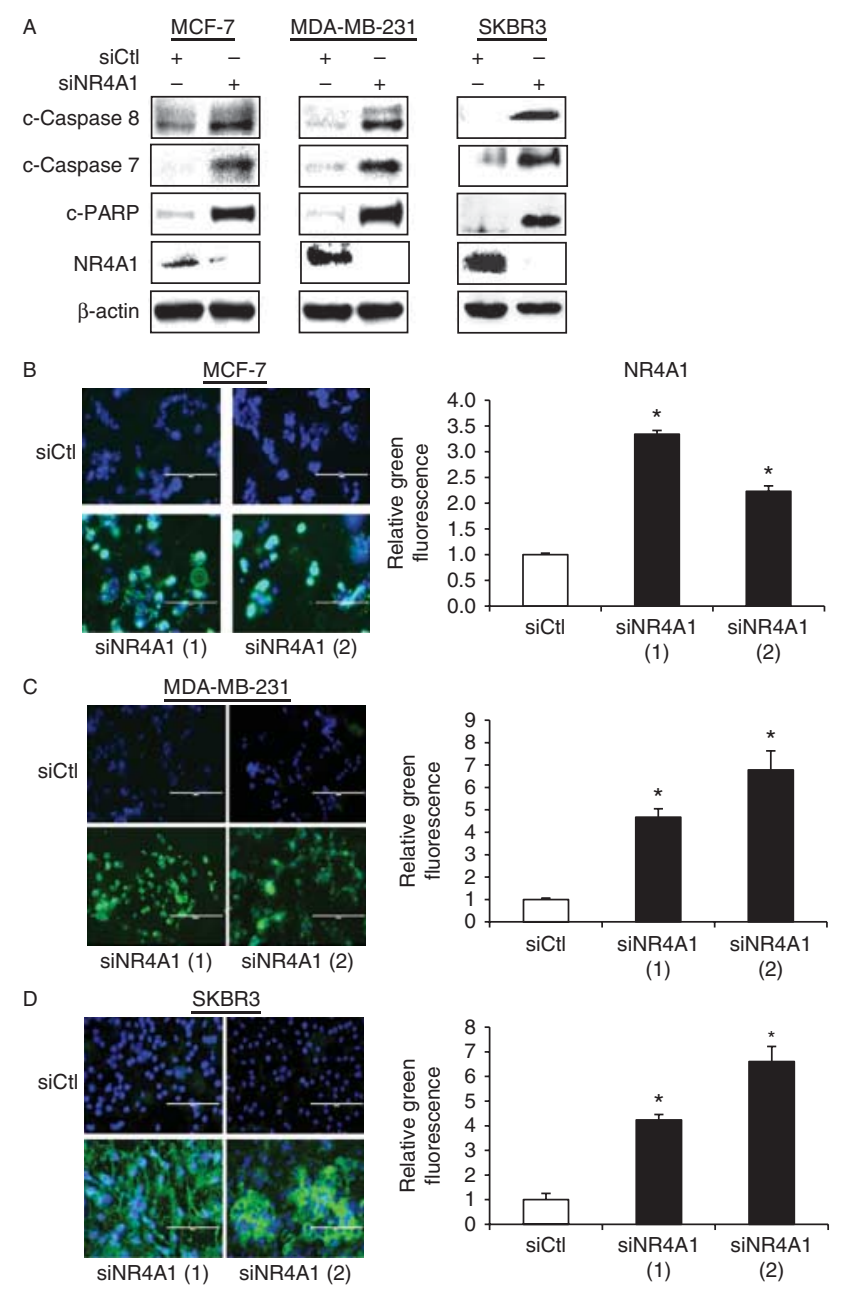

Figure 2

siNR4A1 induces apoptosis in breast cancer cells. (A) Cells were transfected with siNR4A1 and whole cell lysates were analyzed by western blots. Cells were transfected with siNR4A1 (two oligonucleotides) and effects on Annexin V staining in MCF-7 (B), MDA-MB-231 (C), and SKBR3 (D) cells were determined and quantitated. Results (B, $C$ and $D)$ are means \pm s.E.M. for at least three separate determinations and significant $(P<0.05)$ induction of Annexin $V$ staining is indicated $(*)$.

Published by Bioscientifica Ltd. 


\section{Results}

\section{Inhibition of cell proliferation by siNR4A1 and DIM-C-pPhCO $2 \mathrm{Me}$}

The orphan nuclear receptor NR4A1 is overexpressed in ER-positive and ER-negative breast cancer cells (Muscat et al. 2013). The role of this receptor in regulating breast cancer cell growth and survival was investigated by RNAi in ER-positive (MCF-7), ER-negative (MDA-MB-231), and erbB2 (SKBR3) overexpressing breast cancer cell lines. Cells were transfected with two different oligonucleotides against NR4A1 (siNR4A1-1/siNR4A1-2). This resulted in $\geq 50 \%$ growth inhibition in MCF-7 and SKBR3 cells, and $35 \%$ inhibition of MDA-MB-231 cell proliferation (Fig. 1B). The C-DIM compounds with a p-carboxymethylpenyl group (DIM-C-pPhCO ${ }_{2} \mathrm{Me}$ ) and cyano substitutent (DIM-C-pPhCN) have been identified as an NR4A1 antagonists (Lee et al. 2014b). Figure 1C and Supplemental Figure S1A, see section on supplementary data given at the end of this article demonstrate that the former compound significantly inhibits growth of MCF-7, MDA-MB-231 cells after treatment for 24 and $48 \mathrm{~h}$. $\mathrm{IC}_{50}$ values were 20, 19, and $19 \mu \mathrm{M}$ after treatment of MCF-7, MDA-MB-231, and SKBR3 cells, respectively, for $24 \mathrm{~h}$ and 13,19 , and $12 \mu \mathrm{M}$ after treatment for $48 \mathrm{~h}$. In contrast, treatment of nontransformed MCF-10A mammary cells with the high affinity NR4A1 ligand DIM-C-pPhOH $(30 \mu \mathrm{M})$ for 24 or $48 \mathrm{~h}$ did not affect cell proliferation (Supplemental Figure $\mathrm{S} 1 \mathrm{~B})$. Moreover, in an orthotopic model for breast cancer in athymic nude mice using MDA-MB-231 cells treatment with $50 \mathrm{mg} / \mathrm{kg}$ per day and weight compared to the corn oil controls (Fig. 1D). In contrast, after knockdown of NR4A1 in these cells, treatment with DIM-C-pPhCO ${ }_{2} \mathrm{Me}$ resulted in only minimal growth inhibition, which confirms a role for NR4A1 in mediating the growth inhibitory effects of DIM-C-pPhCO ${ }_{2} \mathrm{Me}$ (Fig. 1E).

\section{siNR4A1 and DIM-C-pPhCO 2 Me induce apoptosis and activate growth inhibitory genes/pathways in breast cancer cells}

NR4A1 also regulates pro-survival genes and pathways in pancreatic and lung cancer cells. Figure $2 \mathrm{~A}$ results show transfection of breast cancer cells with siNR4A1 induced cleavage of caspases 8 and 7 as well as PARP cleavage. Moreover, siNR4A1 also induced Annexin V staining MCF-7 (Fig. 2B), MDA-MB-231 (Fig. 2C), and SKBR3 (Fig. 2D) cells confirming that NR4A1 regulated antiapoptotic pathways in these cell lines. Treatment of the cells with DIM-C-pPhCO ${ }_{2} \mathrm{Me}$ for $24 \mathrm{~h}$ also induced cleavage (activation) of caspases 7 and 8 and PARP (Fig. 3A). Similar results were observed for the $p$-cyanophenyl compound (DIM-C-pPhCN) which is an NR4A1 ligand and antagonist in colon cancer cells (Lee et al. 2014b; Supplemental Figure S1C). DIM-C-pPhCO ${ }_{2} \mathrm{Me}$ also enhanced Annexin V staining in MCF-7, MDA-MB-231, and SKBR3 cell lines (Fig. 3B). Moreover, DIM-C-pPhCO ${ }_{2} \mathrm{Me}$-induced apoptosis (PARP cleavage) was reversed after co-administration with the pancaspase inhibitor ZVAD-FMK (Fig. 3C). Thus, both NR4A1 knockdown and NR4A1 antagonists decreased
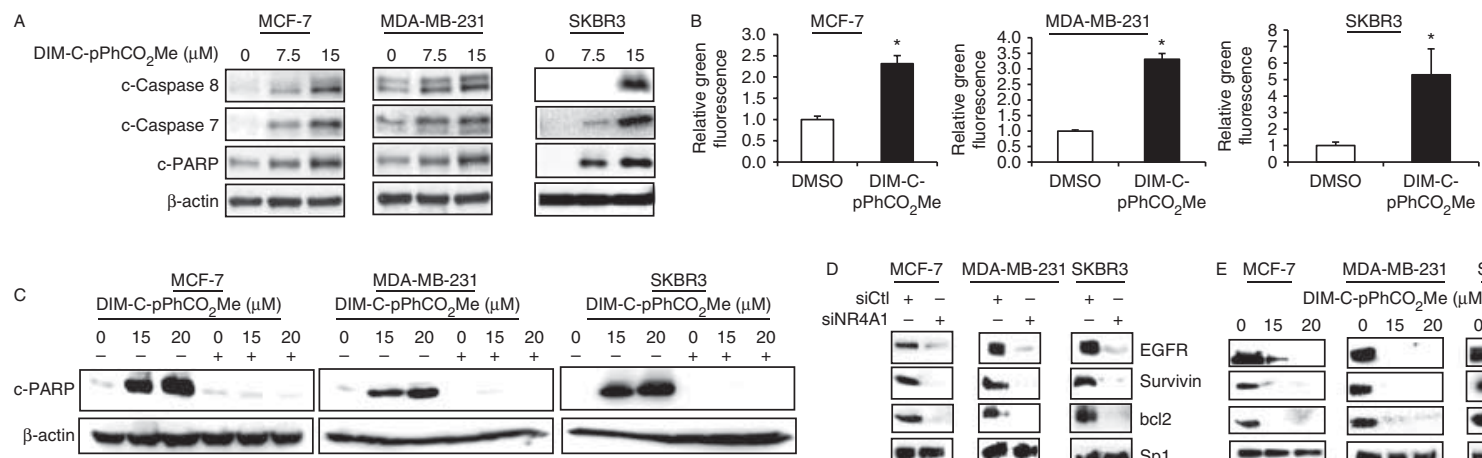

Figure 3

DIM-C-pPhCO 2 Me induces apoptosis and siNR4A1 and DIM-C-pPhCO 2 Me decrease expression of selected genes with GC-rich promoters in breast cancer cells. (A) Cells were treated with DIM-C-pPhCO ${ }_{2} \mathrm{Me}$ for $24 \mathrm{~h}$ and whole cell lysates were analyzed by western blots. (B) MCF-7, MDA-MB-231, and SKBR3 cells were treated with DIM-C-pPhCO $2 \mathrm{Me}$ for $24 \mathrm{~h}$ and Annexin $V$ staining was determined and quantitated. Quantitative results are
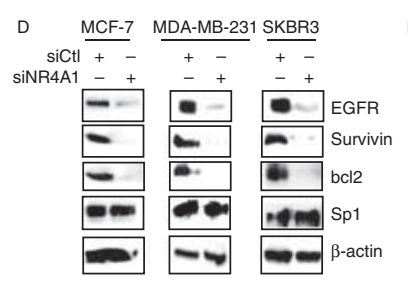
whole cell lysates were analyzed by western blots.

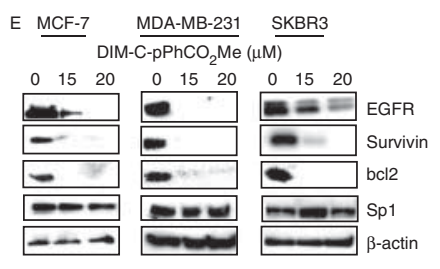

means \pm S.E.M. for three separate determinations and significant $(P<0.05)$ induction of Annexin V is indicated $\left.{ }^{*}\right)$. (C) Cells were treated with DMSO or DIM-C-pPhCO $2 \mathrm{Me}$ alone or in combination with the pan-caspase inhibitor ZVAD-FMK, and PARP cleavage was analyzed by western blots. Cells were transfected with siNR4A1 (D) or treated with DIM-C-pPhCO ${ }_{2} \mathrm{Me}(\mathrm{E})$, and 
breast cancer cell growth and induced apoptosis as well as decreased NR4A1/Sp1-regulated genes as previously described (Lee et al. 2014b). Figure 3D shows that after knockdown of NR4A1 (siNR4A1) in MCF-7, MDA-MB-231, and SKBR3 cells, there was a significant decrease in expression of several Sp1-regulated genes including EGFR, survivin, and bcl-2. However, Sp1 protein levels were unchanged. Similar results were observed in the same cell lines after treatment with the NR4A1 antagonists DIM-C$\mathrm{pPhCO}_{2} \mathrm{Me}$ (Fig. 3D) and DIM-C-pPhCN (Supplemental Figure S1D).

\section{siNR4A1 and DIM-C-pPhCO 2 Me induce ROS and endoplasmic reticulum stress}

It was recently reported that NR4A1 regulates expression of genes such as IDH1 and TXNDC5 that maintain high

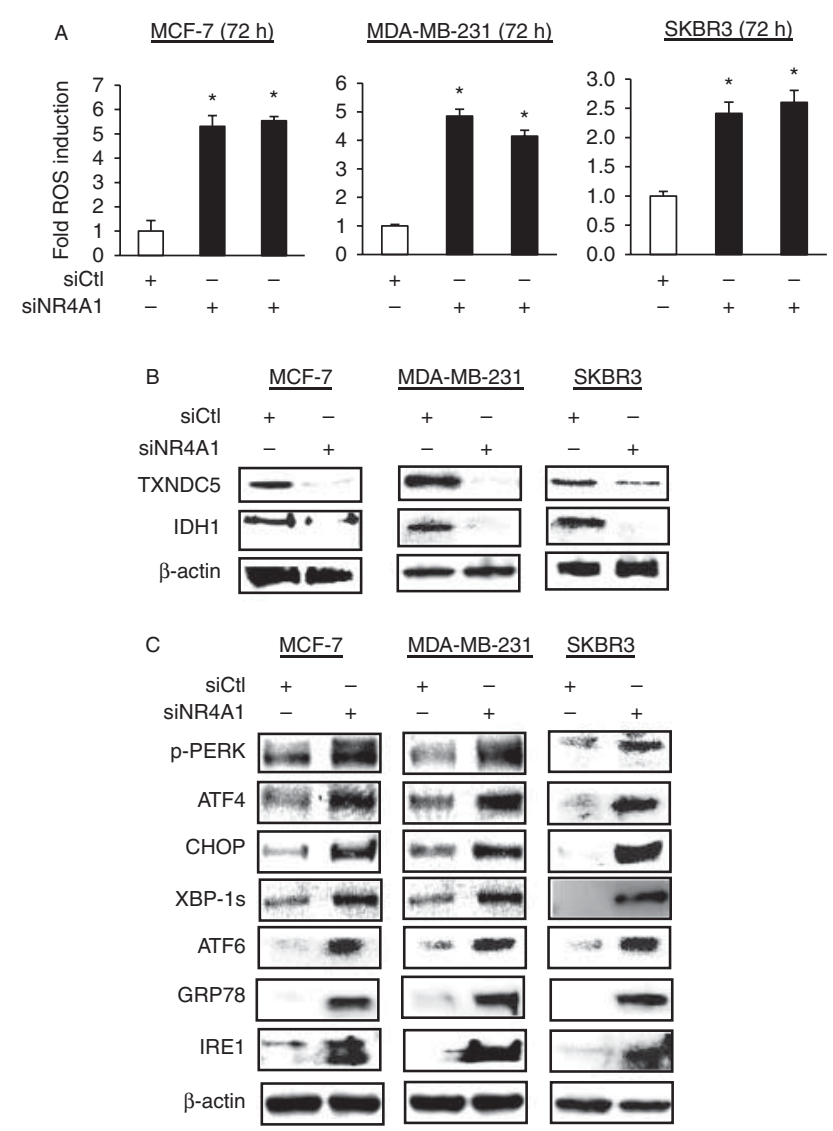

\section{Figure 4}

NR4A1 knockdown induces ROS and cellular stress. (A) Cells were transfected with siNR4A1 or siCtl and ROS was determined. Cells were transfected with siNR4A1 and whole cell lysates were analyzed by western blots for IDH1 or TXNDC5 (B) and ER stress gene products (C). Results in (A) are means \pm s.E.M. for three separate determinations and significant $(P<0.05)$ induction of ROS is indicated $(*)$. levels of reducing equivalents and minimize ROSmediated cellular stress (Lee et al. 2014a,b). Knockdown of NR4A1 by RNAi induced ROS by two- to fourfold in MCF-7, MDA-MB-231, and SKBR3 cells (Fig. 4A). This was accompanied by decreased expression of both TXNDC5 and IDH1 in these cell lines (Fig. 4B). Moreover, after transfection with siNR4A1, we also observed enhanced markers of endoplasmic reticulum (ER) stress including increased phosphorylation of PERK and increased expression of ATF-4, CHOP, GRP78, IRE1, ATF6, and spliced XBP-1 (XBP-1s) in the breast cancer cell lines (Fig. 4C). Treatment of MCF-7, MDA-MB-231, and SKBR3 cells with the NR4A1 antagonist DIM-C-pPhCO ${ }_{2} \mathrm{Me}$ also increased ROS after 12 and $24 \mathrm{~h}$ (Fig. 5A, B and C). This was also accompanied by decreased expression of TXNDC5 and IDH1 (Fig. 5D) as well as induction of markers of ER stress (p-PERK, ATF4, CHOP, ATF6, IRE1, GRP78, and $\mathrm{XBP}-1$; ; Fig. 5E), as previously observed in pancreatic cancer cells (Lee et al. 2014a). The role of ROS in mediating the effects of DIM-C-pPhCO ${ }_{2} \mathrm{Me}$ on activation of stress gene products was investigated in MCF-7, SKBR3, and MDA-MB-231 cells by cotreatment with the antioxidant GSH which reversed induction of the stress genes by the C-DIM/NR4A1 antagonist (Fig. 5F). We also observed that the NR4A1 antagonist DIM-C-pPhCN decreased expression of IDH1 and TXNDC5 in breast cancer cells (Supplemental Figure S1E). In addition, western blot analysis of tumor lysate from control and DIM-C-pPhCO Me-treated mice confirmed that DIM-C- $\mathrm{PPhCO}_{2} \mathrm{Me}$ significantly induced PARP cleavage, decreased expression of Sp-regulated survivin, EGFR and bcl2 gene products and also decreased levels of TXNDC5 and IDH1 (Supplemental Figure S2A and B, see section on supplementary data given at the end of this article).

\section{siNR4A1 and DIM-C-pPhCO ${ }_{2} \mathrm{Me}$ inhibit mTOR signaling}

Transfection of p53-positive MCF-7 cells with siNR4A1 (Fig. 6A) or treatment with DIM-C-pPhCO ${ }_{2} \mathrm{Me}$ (Fig. 6B) induced SESN2, activated AMPK $\alpha$ (phosphorylation), and decreased phosphorylation of mTOR and mTOR downstream genes. Similar results were observed for DIM-CpPhCN (Supplemental Figure S1F). These results are consistent with previous studies in lung cancer cell lines where inactivation of NR4A1 activated p53-dependent activation of SESN2 which in turn activated AMPK $\alpha$ (Lee et al. 2012). Surprisingly, we also observed that transfection of p53-mutant MDA-MB-231 and SKBR3 cells with siNR4A1 induced SESN2, activated AMPK $\alpha$, and inhibited mTOR signaling (Fig. 6C). Similar results were observed

Published by Bioscientifica Ltd. 

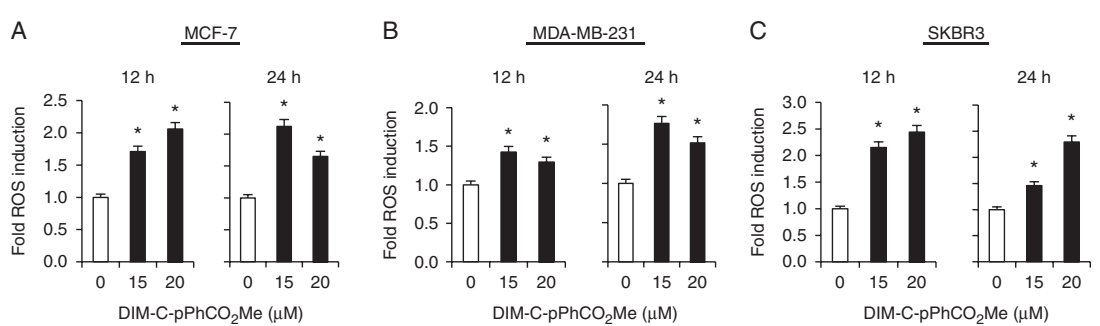

D $\quad$ MCF-7 $\quad$ MDA-MB-231 $\quad$ SKBR3 DIM-C-pPhCO $2 \mathrm{Me}(\mu \mathrm{M})$ DIM-C-pPhCO $2 \mathrm{Me}(\mu \mathrm{M})$

E $\stackrel{\text { MCF-7 }}{\text { MDA-MB-231 }} \underset{\text { DIM-C-pPhCO } 2 M e(\mu M)}{-} \underline{\text { SKBR3 }}$


\section{Figure 5}

DIM-C-pPhCO 2 Me induces ROS and stress. MCF-7 (A), MDA-MB-231 (B), and SKBR3 (C) cells were treated with DIM-C-pPhCO ${ }_{2} \mathrm{Me}$ and ROS was determined after 12 or $24 \mathrm{~h}$. Cells were treated with DIM-C-pPhCO $\mathrm{Me}^{\mathrm{Me}}$ and whole cell lysates were analyzed by western blots for expression of TXNDC5 (D), IDH1 (D), and stress gene products (E). (F) Cells were treated with

after treatment with DIM-C-pPhCO ${ }_{2} \mathrm{Me}$. We also recently observed similar results in a p53-mutant renal adenocarcinoma cell line (Hedrick et al. 2015) which showed that induction of SESN2 was ROS-dependent. This was consistent with previous reports showing that SESN2 was an oxygen-sensing gene (Budanov et al. 2002). Figure 6D shows that induction of SESN2 and activation of AMPK $\alpha$ in MDA-MB-231 and SKBR3 cells after treatment with DIM-C-pPhCO $\mathrm{C}_{2} \mathrm{Me}$ was inhibited after cotreatment with GSH. These data are consistent with the induction of ROS in these cell lines after transfection with siNR4A1 (Fig. 4A) or treatment with DIM-C-pPhCO ${ }_{2} \mathrm{Me}$ (Fig. 5A, B and C). Thus, results of this study demonstrate that NR4A1 regulates multiple pro-oncogenic pathways in breast cancer cells. Immunostaining for NR4A1 demonstrates that the receptor is nuclear and remains in the nucleus after treatment with an antagonist (Supplemental Figure S3, see section on supplementary data given at the end of this article). Thus, C-DIM/NR4A1 antagonists inhibit multiple nuclear NR4A1-mediated genes/pathways (Fig. 1A) and represent a new class of mechanism-based drugs for breast cancer chemotherapy.
DMSO, DIM-C-pPhCO ${ }_{2}$ Me alone or in combination with GSH, and whole cell lysates were analyzed for stress gene products by western blots. Results (A) are expressed as means \pm S.E.M. for three separate determinations and significant $(P<0.05)$ induction of ROS is indicated $\left(^{*}\right)$.

\section{Discussion}

NR4A1 exhibits pro-oncogenic activity in cancer cell lines derived from solid tumors and is overexpressed in tumors from lung, pancreatic, and colon cancer patients (Chintharlapalli et al. 2005, Cho et al. 2010, Lee et al. 2010, 2012, 2014a, Wu et al. 2011). Moreover, in lung cancer patients, high expression of NR4A1 is a prognostic indicator for decreased survival (Lee et al. 2012). Similar results were recently reported for the expression and prognostic activity of NR4A1 in breast cancer patients. It was also observed that NR4A1 was one of only a few nuclear receptors overexpressed in patients with both ER-positive and ER-negative breast tumors (Muscat et al. 2013, Zhou et al. 2014). Since an early report indicated that NR4A1 is more highly expressed in early- vs late-stage aggressive breast tumors and exhibited some tumor suppressor-like activity (Alexopoulou et al. 2010), we investigated the function of NR4A1 in three different breast cancer cell lines by RNAi and treatment with NR4A1 antagonists. We have recently demonstrated that several C-DIMs including the p-hydroxy, carbomethoxy, cyano,

Published by Bioscientifica Ltd 

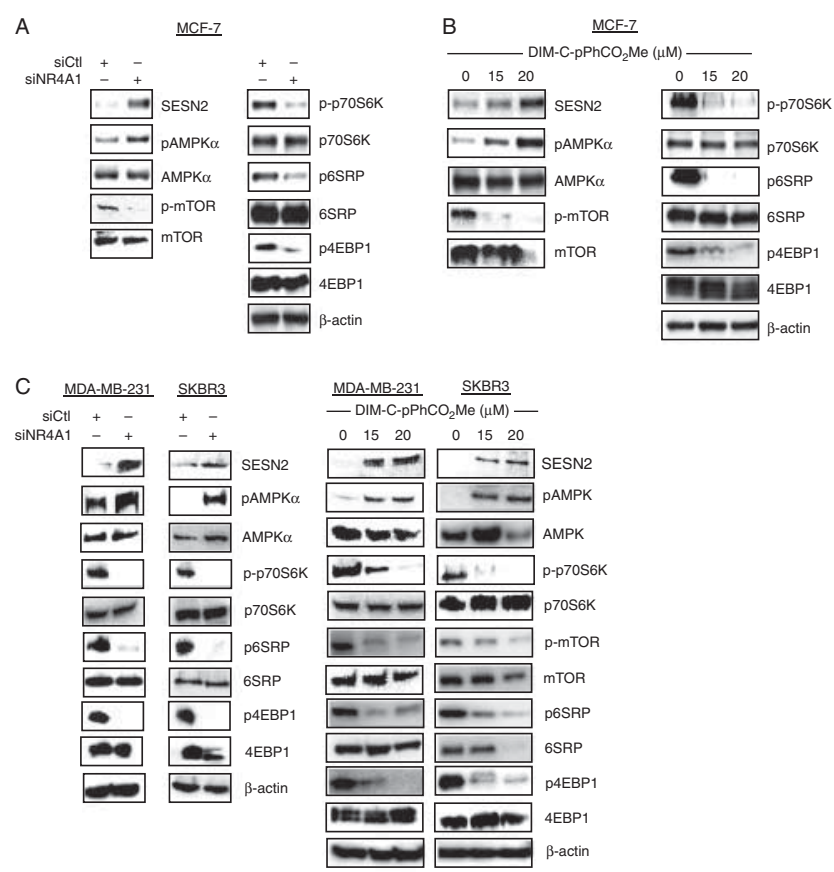

D

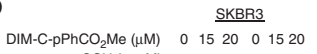

MDA-MB-231 $0 \longdiv { 1 5 2 0 0 1 5 2 0 }$ GSH (5 mM)


Figure 6

NR4A1 knockdown and DIMC-C-pPhCO 2 Me inhibit mTOR. MCF-7 cells were transfected with siNR4A1 (A) or treated with DIM-C-pPhCO ${ }_{2} \mathrm{Me}(\mathrm{B})$ and whole cell lysates were analyzed by western blots for mTOR pathway gene products. (C) MDA-MB-231 and SKBR3 cells were transfected with siNR4A1 or treated with DIM-C-pPhCO $2 \mathrm{Me}$, and whole cell lysates were analyzed for mTOR pathway genes by western blots. (D) MDA-MB-231 and SKBR3 cells were treated with DMSO, DIM-C-pPhCO ${ }_{2} \mathrm{Me}$ alone or in combination with GSH and, after $24 \mathrm{~h}$ whole cell lysates were analyzed by western blots for sestrin 2 and AMPK $\alpha$.

and bromophenyl analogs directly bind the ligand binding domain of NR4A1 and exhibit NR4A1 antagonist activity in colon cancer cells (Lee et al. 2014b). The $p$-carbomethyoxyphenyl analog (DIM-C-pPhCO ${ }_{2} \mathrm{Me}$ ) and to a lesser extent DIM-C-pPhCN were used as prototypical C-DIMs/NR4A1 antagonists for investigating the anticancer activities of NR4A1 antagonists in breast cancer cells with a focus on inhibition of three previously identified pro-oncogenic NR4A1-regulated pathways (Fig. 1A).

Initial studies investigated the role of NR4A1 in the growth of three prototypical breast cancer cell lines (ER-positive MCF-7, erbB2 overexpressing SKBR3, and triple negative MDA-MB-231 cells). Knockdown of NR4A1 using two different oligonucleotides significantly decreased proliferation of all three breast cancer cell lines and similar growth inhibitory effects were observed for DIM-C-pPhCO ${ }_{2} \mathrm{Me}$ (Fig. 1B and C). Moreover, after knockdown of NR4A1 in MCF-7, MDA-MB-231, and SKBR3 cells, treatment with DIM-C-pPhCO${ }_{2} \mathrm{Me}$ had minimal effects (Fig. 1D), suggesting that the growth inhibitory effects of DIM-C-pPhCO ${ }_{2} \mathrm{Me}$ were primarily NR4A1-dependent. The effects of NR4A1 knockdown or treatment with DIM-C-pPhCO ${ }_{2} \mathrm{Me}$ on several markers of apoptosis, including cleavage of caspases 7 and 8 and PARP as well as induction of Annexin $\mathrm{V}$ staining, were also determined in the breast cancer cell lines (Figs 2 and 3). These results demonstrate that NR4A1 regulates pathways that contribute to the growth and survival of breast cancer cell. This parallels the functions previously observed for this receptor in pancreatic, lung, and colon cancer cells (Lee et al. 2010, 2012, 2014a,b).

NR4A1 binds and inactivates p53 (Zhao et al. 2006), whereas knockdown of NR4A1 or treatment of p53 WT lung cancer cells with an NR4A1 antagonist or transfection with siNR4A1 results in activation of p53 and induction of SESN2 which activates AMPK $\alpha$ and inhibits the mTOR pathway (Lee et al. 2012). mTOR pathway inhibitors have been extensively developed for cancer chemotherapy (Baselga et al. 2012, Ciruelos Gil 2014). C-DIM/NR4A1 antagonists represent a new class of mTOR inhibitors which block NR4A1-regulated mTOR activation in cancer cells expressing WT p53 (Lee et al. 2012). Results illustrated in Fig. 6A and $\mathrm{B}$ show that both siNR4A1 and DIM$\mathrm{C}$-pPhCO $\mathrm{CP}_{2} \mathrm{Me}$ inhibited mTOR pathway in MCF-7 breast cancer cells that express WT p53. In p53 WT lung cancer cells, siNR4A1 and NR4A1 antagonists also induced SESN2 which activates AMPK $\alpha$ and inhibits mTOR, whereas this is not observed in lung cancer cells expressing mutant p53 (Lee et al. 2012). Interestingly, DIM-C-pPhCO ${ }_{2} \mathrm{Me}$ and siNR4A1 also induced SESN2 and inhibited mTOR in p53 mutant SKBR3 and MDA-MB-231 cells (Fig. 6C). The DIM-C-pPhCO ${ }_{2}$ Me-mediated induction of SESN2 and activation of AMPK $\alpha$ were reversed after cotreatment with GSH (Fig. 6D). These results are consistent with induction of ROS by DIM-C-pPhCO ${ }_{2} \mathrm{Me}$ (Fig. 5A, B and C) and the fact that SESN2 is an oxygen-sensing gene that is induced by ROS (Budanov et al. 2002).

Like other nuclear receptors, NR4A1 interacts with the Sp1 transcription factor bound to GC-rich sites to activate survivin and other anti-apoptotic/growth promoting genes. siNR4A1 or treatment with a NR4A1 antagonist decreases expression of these genes (Liu \& Simpson 1999, Pipaon et al. 1999, Suzuki et al. 1999, Lu et al. 2000, Shimada et al. 2001, Sugawara et al. 2002). Figure 3C and D

Published by Bioscientifica Ltd. 
show that siNR4A1 or treatment with DIM-C-pPhCO ${ }_{2} \mathrm{Me}$ decreased expression of survivin, bcl2 and EGFR in MDAMB-231, MCF-7, and SKBR3 cells. However, Sp protein levels were unchanged. Molecular analysis of NR4A1dependent regulation of survivin showed that in pancreatic cancer cells, NR4A1 and p300 cooperatively activated survivin expression by interacting with $\mathrm{Sp} 1$ bound to the proximal GC-rich region of the survivin promoter (Lee et al. 2010). Regulation of growth-promoting and survival genes which contain GC-rich promoters by NR4A1 is consistent with the growth inhibitory and apoptotic effects of siNR4A1 and C-DIM/NR4A1 antagonists on breast cancer cells and tumors. It is comparable to that observed in lung, colon and pancreatic cancer cell lines (Lee et al. 2010, 2012, 2014a,b).

A recent study showed that NR4A1 maintains low levels of oxidative and ER stress in pancreatic cancer cells by regulating expression of TXNDC5 and IDH1 which maintain cellular levels of reducing equivalents (Lee et al. 2014a). DIM-C-pPhCO ${ }_{2} \mathrm{Me}$ or siNR4A1 decreased expression of TXNDC5 and IDH1 in breast tumors (in vivo; Supplemental Figure S2) and in MCF-7, MDA-MB-231, and SKBR3 cells. This was accompanied by increased levels of ROS and induction of markers of ER stress (Figs 4 and 5). These results are consistent with previous studies in pancreatic cancer cells. There is also emerging evidence that both TXNDC5 and IDH1 are overexpressed in breast cancer cells and tumors (Xu et al. 2010, Chang et al. 2013). Their expression and functions in breast cancer cells are currently being investigated.

In summary, results of this study are consistent with a pro-oncogenic role for NR4A1 in breast cancer as an important regulator of cell growth and survival. NR4A1regulated pro-oncogenic pathways and genes are similar to those observed in pancreatic, lung, and colon cancer cells and tumors (Lee et al. 2010, 2012, 2014a,b). This study also demonstrated the effectiveness of the NR4A1 antagonists DIM-C-pPhCO ${ }_{2} \mathrm{Me}$ and DIM-C-pPhCN as inhibitors of breast cancer cell and tumor growth as well as survival. Current structure-activity studies are focused on identifying the most effective C-DIM/NR4A1 antagonists for future clinical applications in breast cancer chemotherapy, including the inhibition of breast invasion (Zhou et al. 2014) through inhibition of nuclear NR4A1 by receptor antagonists.

\section{Supplementary data}

This is linked to the online version of the paper at http://dx.doi.org/10.1530/ ERC-15-0063.
Declaration of interest

The authors declare that there is no conflict of interest that could be perceived as prejudicing the impartiality of the research reported.

\section{Funding}

Funding was provided by DOD-CDRMP (BC103116; M Singh), NIEHS (P30-ES023512; S Safe), Texas AgriLife Research, and the Sid Kyle Endowment (S Safe)

\section{References}

Alexopoulou AN, Leao M, Caballero OL, Da Silva L, Reid L, Lakhani SR, Simpson AJ, Marshall JF, Neville AM \& Jat PS 2010 Dissecting the transcriptional networks underlying breast cancer: NR4A1 reduces the migration of normal and breast cancer cell lines. Breast Cancer Research 12 R51. (doi:10.1186/bcr2610)

Baselga J, Campone M, Piccart M, Burris HA III, Rugo HS, Sahmoud T, Noguchi S, Gnant M, Pritchard KI, Lebrun F et al. 2012 Everolimus in postmenopausal hormone-receptor-positive advanced breast cancer. New England Journal of Medicine 366 520-529. (doi:10.1056/ NEJMoa1109653)

Bras A, Albar JP, Leonardo E, de Buitrago GG \& Martinez AC 2000 Ceramide-induced cell death is independent of the Fas/Fas ligand pathway and is prevented by Nur77 overexpression in A20 B cells. Cell Death and Differentiation 7 262-271. (doi:10.1038/sj.cdd.4400653)

Budanov AV, Shoshani T, Faerman A, Zelin E, Kamer I, Kalinski H, Gorodin S, Fishman A, Chajut A, Einat P et al. 2002 Identification of a novel stress-responsive gene Hi95 involved in regulation of cell viability. Oncogene 21 6017-6031. (doi:10.1038/sj.onc.1205877)

Chang X, Xu B, Wang L, Wang Y, Wang Y \& Yan S 2013 Investigating a pathogenic role for TXNDC5 in tumors. International Journal of Oncology 43 1871-1884. (doi:10.3892/ijo.2013.2123)

Chintharlapalli S, Burghardt R, Papineni S, Ramaiah S, Yoon K \& Safe S 2005 Activation of Nur77 by selected 1,1-bis(3'-indolyl)-1( $p$-substituted phenyl)methanes induces apoptosis through nuclear pathways. Journal of Biological Chemistry $28024903-24914$. (doi:10.1074/jbc.M500107200)

Cho SD, Lee SO, Chintharlapalli S, Abdelrahim M, Khan S, Yoon K, Kamat AM $\&$ Safe S 2010 Activation of nerve growth factor-induced $\mathrm{B} \alpha$ by methylene-substituted diindolylmethanes in bladder cancer cells induces apoptosis and inhibits tumor growth. Molecular Pharmacology 77 396-404. (doi:10.1124/mol.109.061143)

Ciruelos Gil EM 2014 Targeting the PI3K/AKT/mTOR pathway in estrogen receptor-positive breast cancer. Cancer Treatment Reviews 40 862-871. (doi:10.1016/j.ctrv.2014.03.004)

Ferlini C, Cicchillitti L, Raspaglio G, Bartollino S, Cimitan S, Bertucci C, Mozzetti S, Gallo D, Persico M, Fattorusso C et al. 2009 Paclitaxel directly binds to Bcl-2 and functionally mimics activity of Nur77. Cancer Research 69 6906-6914. (doi:10.1158/0008-5472.CAN-09-0540)

Hedrick E, Lee SO, Kim G, Abdelrahim M, Jin UH, Safe S \& Abudayyeh A 2015 Nuclear receptor 4A1 (NR4A1) as a drug target for renal cell adenocarcinoma. PLOS ONE 10 e0128308. (doi:10.1371/journal.pone. 0128308)

Kolluri SK, Bruey-Sedano N, Cao X, Lin B, Lin F, Han YH, Dawson MI \& Zhang XK 2003 Mitogenic effect of orphan receptor TR3 and its regulation by MEKK1 in lung cancer cells. Molecular and Cellular Biology 23 8651-8667. (doi:10.1128/MCB.23.23.8651-8667.2003)

Kolluri SK, Zhu X, Zhou X, Lin B, Chen Y, Sun K, Tian X, Town J, Cao X, Lin F et al. 2008 A short Nur77-derived peptide converts Bcl-2 from a protector to a killer. Cancer Cell 14 285-298. (doi:10.1016/j.ccr.2008. 09.002)

Published by Bioscientifica Ltd. 
Lee SO, Chintharlapalli S, Liu S, Papineni S, Cho SD, Yoon K \& Safe S 2009 p21 expression is induced by activation of nuclear nerve growth factorinduced $\mathrm{B} \alpha$ (Nur77) in pancreatic cancer cells. Molecular Cancer Research 7 1169-1178. (doi:10.1158/1541-7786.MCR-08-0473)

Lee SO, Abdelrahim M, Yoon K, Chintharlapalli S, Papineni S, Kim K, Wang H \& Safe S 2010 Inactivation of the orphan nuclear receptor TR3/Nur77 inhibits pancreatic cancer cell and tumor growth. Cancer Research 70 6824-6836. (doi:10.1158/0008-5472.CAN-10-1992)

Lee SO, Li X, Khan S \& Safe S 2011 Targeting NR4A1 (TR3) in cancer cells and tumors. Expert Opinion on Therapeutic Targets 15 195-206. (doi:10. 1517/14728222.2011.547481)

Lee SO, Andey T, Jin UH, Kim K, Singh M \& Safe S 2012 The nuclear receptor TR3 regulates mTORC1 signaling in lung cancer cells expressing wildtype p53. Oncogene 31 3265-3276. (doi:10.1038/onc.2011.504)

Lee SO, Jin UH, Kang JH, Kim SB, Guthrie AS, Sreevalsan S, Lee JS \& Safe S $2014 a$ The orphan nuclear receptor NR4A1 (Nur77) regulates oxidative and endoplasmic reticulum stress in pancreatic cancer cells. Molecular Cancer Research 12 527-538. (doi:10.1158/1541-7786.MCR13-0567)

Lee SO, Li X, Hedrick E, Jin UH, Tjalkens RB, Backos DS, Li L, Zhang Y, Wu Q \& Safe S 2014b Diindolylmethane analogs bind NR4A1 and are NR4A1 antagonists in colon cancer cells. Molecular Endocrinology $\mathbf{2 8}$ 1729-1739. (doi:10.1210/me.2014-1102)

Li H, Kolluri SK, Gu J, Dawson MI, Cao X, Hobbs PD, Lin B, Chen G, Lu J, Lin F et al. 2000 Cytochrome $c$ release and apoptosis induced by mitochondrial targeting of nuclear orphan receptor TR3. Science $\mathbf{2 8 9}$ 1159-1164. (doi:10.1126/science.289.5482.1159)

Lin B, Kolluri SK, Lin F, Liu W, Han YH, Cao X, Dawson MI, Reed JC \& Zhang XK 2004 Conversion of Bcl-2 from protector to killer by interaction with nuclear orphan receptor Nur77/TR3. Cell 116 527-540. (doi:10.1016/S0092-8674(04)00162-X)

Liu Z \& Simpson ER 1999 Molecular mechanism for cooperation between Sp1 and steroidogenic factor-1 (SF-1) to regulate bovine CYP11A gene expression. Molecular and Cellular Endocrinology 153 183-196. (doi:10. 1016/S0303-7207(99)00036-2)

Liu JJ, Zeng HN, Zhang LR, Zhan YY, Chen Y, Wang Y, Wang J, Xiang SH, Liu WJ, Wang WJ et al. 2010 A unique pharmacophore for activation of the nuclear orphan receptor Nur77 in vivo and in vitro. Cancer Research 70 3628-3637. (doi:10.1158/0008-5472.CAN-09-3160)

Lu S, Jenster G \& Epner DE 2000 Androgen induction of cyclin-dependent kinase inhibitor $\mathrm{p} 21$ gene: role of androgen receptor and transcription factor Sp1 complex. Molecular Endocrinology 14 753-760. (doi:10.1210/ mend.14.5.0461)

Maxwell MA \& Muscat GE 2006 The NR4A subgroup: immediate early response genes with pleiotropic physiological roles. Nuclear Receptor Signaling 4 e002. (doi:10.1621/nrs.04002)

Muscat GE, Eriksson NA, Byth K, Loi S, Graham D, Jindal S, Davis MJ, Clyne C, FunderJW, Simpson ER etal. 2013 Research resource: Nuclear receptors as transcriptome: discriminant and prognostic value in breast cancer. Molecular Endocrinology 27 350-365. (doi:10.1210/me.2012-1265)

Pearen MA \& Muscat GE 2010 Minireview: Nuclear hormone receptor 4A signaling: implications for metabolic disease. Molecular Endocrinology 24 1891-1903. (doi:10.1210/me.2010-0015)
Pipaon C, Tsai SY \& Tsai MJ 1999 COUP-TF upregulates NGFI-A gene expression through an Sp1 binding site. Molecular and Cellular Biology 19 2734-2745.

Shimada J, Suzuki Y, Kim SJ, Wang PC, Matsumura M \& Kojima S 2001 Transactivation via RAR/RXR-Sp1 interaction: characterization of binding between Sp1 and GC box motif. Molecular Endocrinology 15 1677-1692. (doi:10.1210/mend.15.10.0707)

Sugawara A, Uruno A, Kudo M, Ikeda Y, Sato K, Taniyama Y, Ito S \& Takeuchi K 2002 Transcription suppression of thromboxane receptor gene by peroxisome proliferator-activated receptor- $\gamma$ via an interaction with Sp1 in vascular smooth muscle cells. Journal of Biological Chemistry 277 9676-9683. (doi:10.1074/jbc.M104560200)

Suzuki Y, Shimada J, Shudo K, Matsumura M, Crippa MP \& Kojima S 1999 Physical interaction between retinoic acid receptor and Sp1: mechanism for induction of urokinase by retinoic acid. Blood 93 4264-4276.

Uemura H \& Chang C 1998 Antisense TR3 orphan receptor can increase prostate cancer cell viability with etoposide treatment. Endocrinology 139 2329-2334. (doi:10.1210/endo.139.5.5969)

Wang WJ, Wang Y, Chen HZ, Xing YZ, Li FW, Zhang Q, Zhou B, Zhang HK, Zhang J, Bian XL et al. 2014 Orphan nuclear receptor TR3 acts in autophagic cell death via mitochondrial signaling pathway. Nature Chemical Biology 10 133-140. (doi:10.1038/nchembio.1406)

Wu H, Lin Y, Li W, Sun Z, Gao W, Zhang H, Xie L, Jiang F, Qin B, Yan T et al. 2011 Regulation of Nur77 expression by $\beta$-catenin and its mitogenic effect in colon cancer cells. FASEB Journal 25 192-205. (doi:10.1096/ fj.10-166462)

Xu SG, Yan PJ \& Shao ZM 2010 Differential proteomic analysis of a highly metastatic variant of human breast cancer cells using two-dimensional differential gel electrophoresis. Journal of Cancer Research and Clinical Oncology 136 1545-1556. (doi:10.1007/s00432-010-0812-0)

Zeng JZ, Sun DF, Wang L, Cao X, Qi JB, Yang T, Hu CQ, Liu W \& Zhang XK 2006 Hypericum sampsonii induces apoptosis and nuclear export of retinoid X receptor- $\alpha$. Carcinogenesis 27 1991-2000. (doi:10.1093/ carcin/bgl046)

Zhan Y, Du X, Chen H, Liu J, Zhao B, Huang D, Li G, Xu Q, Zhang M, Weimer BC et al. 2008 Cytosporone B is an agonist for nuclear orphan receptor Nur77. Nature Chemical Biology 4 548-556. (doi:10.1038/ nchembio.106)

Zhan YY, Chen Y, Zhang Q, Zhuang JJ, Tian M, Chen HZ, Zhang LR, Zhang HK, He JP, Wang WJ et al. 2012 The orphan nuclear receptor Nur77 regulates LKB1 localization and activates AMPK. Nature Chemical Biology 8 897-904. (doi:10.1038/nchembio.1069)

Zhang XK 2007 Targeting Nur77 translocation. Expert Opinion on Therapeutic Targets 11 69-79. (doi:10.1517/14728222.11.1.69)

Zhao BX, Chen HZ, Lei NZ, Li GD, Zhao WX, Zhan YY, Liu B, Lin SC \& Wu Q 2006 p53 mediates the negative regulation of MDM2 by orphan receptor TR3. EMBO Journal 25 5703-5715. (doi:10.1038/sj.emboj. 7601435)

Zhou F, Drabsch Y, Dekker TJ, de Vinuesa AG, Li Y, Hawinkels LJ, Sheppard KA, Goumans MJ, Luwor RB, de Vries CJ et al. 2014 Nuclear receptor NR4A1 promotes breast cancer invasion and metastasis by activating TGF- $\beta$ signalling. Nature Communications 53388. (doi:10.1038/ncomms4388)

Received in final form 28 July 2015

Accepted 30 July 2015

Made available online as an Accepted Preprint

30 July 2015 http://erc.endocrinology-journals.org DOI: $10.1530 / E R C-15-0063$
(C) 2015 Society for Endocrinology Printed in Great Britain
Published by Bioscientifica Ltd 